\title{
Micromachined electromagnetic scanning mirrors
}

\author{
Raanan A. Miller, MEMBER SPIE \\ Yu-Chong Tai \\ California Institute of Technology \\ Electrical Engineering, MS 136-93 \\ Pasadena, California 91125 \\ E-mail: ram@touch.caltech.edu
}

\begin{abstract}
We report a new magnetic MEMS technology that enables many electromagnetic MEMS devices. This new technology combines magnetic thin films and silicon bulk micromachining. Its use is demonstrated by two types of millimeter-sized analog scanning mirrors that are capable of delivering deflection angles exceeding $60 \mathrm{deg}$. Details include the design, fabrication, operation, as well as a complete electromechanical model of the mirrors. In addition, the use of the mirrors is further manifested in a holographic data storage system where hundreds of holograms have been successfully stored and retrieved. (C) 1997 Society of Photo-Optical Instrumentation Engineers. [S0091-3286(97)01005-2]
\end{abstract}

Subject terms: micro-opto-electro-mechanical systems; electromagnetic actuation; laser scanning mirror; holographic data storage; permalloy.

Paper MEM-10 received Nov. 8, 1996; revised manuscript received Jan. 14, 1997; accepted for publication Jan. 24, 1997.

\section{Introduction}

Recent developments in silicon micromachining have enabled many novel micro-electro-mechanical systems (MEMSs). ${ }^{1}$ These systems, however, are mainly developed for microsensors; considerably less work has been done on microactuators. Even among the few applications to actuators, the majority of effort has been on electrostatic and piezoelectric actuation. Nevertheless, we think electromagnetic actuation is very promising, and magnetic actuation has many advantages over both electrostatic and piezoelectric actuation. For example, achievable magnetic forces in a microactuator can be both attractive and repulsive. They can also be large in magnitude (on the order of millinewtons) and effective over a long range (on the order of millimeters). ${ }^{2}$ The major disadvantage of magnetic actuation is the lack of a mature technology. This is mainly because magnetic actuation requires more complicated components, such as coils and magnetic core materials.

Here we report our efforts to develop a new magnetic MEMS technology, which enables many novel magnetic MEMS devices. Our approach is to integrate magnetic thin films with bulk silicon micromachining. As a demonstration, we have developed two types of small-size, low-cost, highly accurate, large-angle-of-deflection MEMS laserscanning mirrors. These devices can be used in many applications, ranging from bar-code scanners to scanning mirrors in holographic data storage systems. The two types of actuators are: (1) mirrors with permalloy $\left(\mathrm{Ni}_{90} \mathrm{Fe}_{10}\right)$ only, and (2) mirrors with both permalloy and copper coils. The two devices have different advantages, and the choice of them depends on the application. The type-1 mirrors can be controlled by an external magnetic field, so they do not experience thermal heating effects. The type- 2 mirrors also require an external field but can be controlled using a coil current. This feature is especially useful if individual addressability is necessary when many mirrors are used in an array.

\section{Design}

The mechanical structure of the permalloy-only (type-1) mirror (Fig. 1) consists of a single-crystalline silicon plate $\left(4 \times 4 \times 0.04 \mathrm{~mm}^{3}\right)$ tethered to the bulk silicon substrate by two serpentine springs. A $3-\mathrm{mm} \times 3-\mathrm{mm}$ permalloy layer is located on the bottom side of the plate and is used to induce the magnetic actuation. An additional permalloy mesh structure sits on the top side of the plate and constrains the mirror to deflections only above the substrate. Here the top surface of the plate framed by the mesh is the mirror reflector. The type-2 mirror design then includes a 30-turn copper coil in addition to the permalloy layer. This mirror (Fig. 2) has the same silicon plate and springs as the type-1 mirror, but its bottom mirrorlike surface (single-crystal silicon) serves as the reflector and on its top surface sit the 30-turn copper coil and the thin-film permalloy layer. Both the coil and the permalloy can be used to provide the actuation forces.

\section{Fabrication}

The fabrication steps for the type-1 mirror (Fig. 3) start with the bulk micromachining of a $40-\mu$ m-thick epitaxial silicon membrane by anisotropically etching the bulk silicon down to a boron etch-stop layer. The boron layer is then removed, and an oxide layer is grown. A Ti/Cu seedlayer is next evaporated on the bottom side of the silicon plate. A photoresist mold is formed, and $20 \mu \mathrm{m}$ of permalloy is deposited. The photoresist and seed layer are then removed. Next a Ti/Cu seedlayer is evaporated on the top side of the mirror, and a $10-\mu \mathrm{m}$ permalloy mesh structure is electroplated. This mesh extends beyond the intended (4 $\times 4 \mathrm{~mm}^{2}$ ) border of the plate. The mirror is then freed from the substrate in two steps. First, in the areas outside the plate region the permalloy mesh is detached from the silicon substrate by etching away the oxide layer with an HF enchant. Then, the plate and springs are lithographically patterned on the membrane and free-released by reactive- 

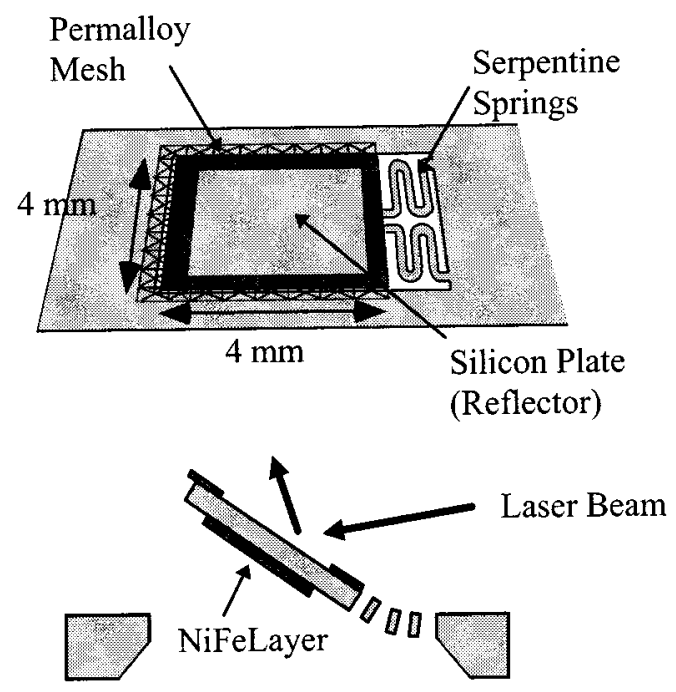

Fig. 1 Schematic top view and cross-sectional view (deflected) of permalloy-only (type-1) mirror.

ion etching (RIE). In this RIE step, the silicon under the permalloy mesh is also etched so as to free the mirror from the substrate. The mesh remains overhanging the edge of the freed plate, and serves two purposes. First, it constrains the mirror to deflections above the plane. Second, the mesh improves the shock resistance of the mirrors, which allows mirrors with softer springs (hence larger deflections at lower magnetic fields). Finally, a thin $(200 \mathrm{~nm})$ layer of aluminum is evaporated on the top reflector to serve as the mirror surface.

The fabrication steps for the type-2 mirror (Fig. 4) also involve the formation of a $40 \mu \mathrm{m}$ membrane as described previously. A $\mathrm{Ti} / \mathrm{Cu}$ seedlayer is however evaporated on the top side of the plate. A photoresist mold is formed, and
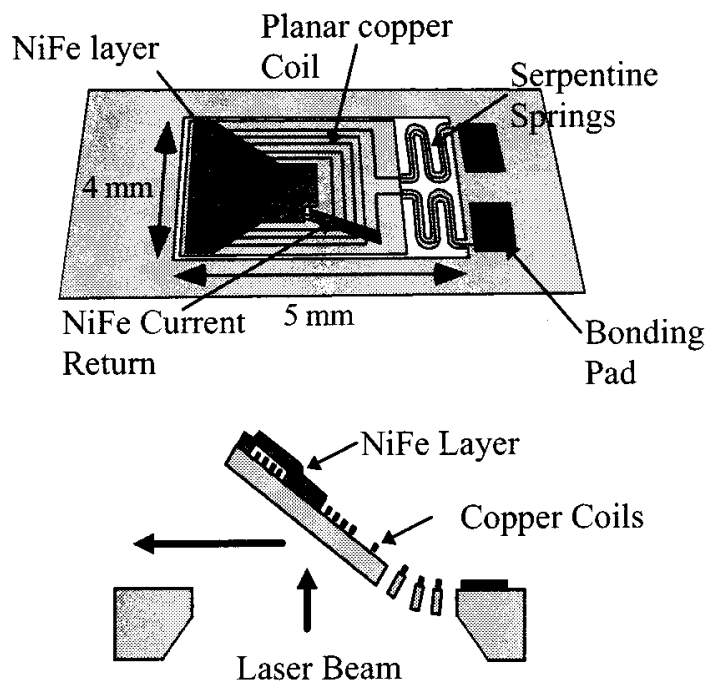

Fig. 2 Schematic top view and cross-sectional view (deflected) of type-2 MEMS mirror. a 30-turn planar copper coil is electroplated ( $9 \mu \mathrm{m}$ thick). Next an insulating photoresist layer is coated, patterned, and hard-baked. A Ti/Cu seedlayer is again evaporated, a photoresist mold is formed, and an $11-\mu$ m-thick permalloy layer electroplated. Here, a small piece of permalloy (as shown in Fig. 2) serves as a current return path when current is provided to the coil. Finally, a thick layer of photoresist is coated and the spring and plate structures are photolithographically defined. The exposed oxide layer is removed in buffered hydrofluoric acid (BHF), and the silicon beneath is reactive-ion-etched to free-release the mirror.

\section{Mirror Operation}

There are two modes of magnetic actuation used in our mirrors. The first is permalloy actuation, and the second is coil actuation. Permalloy actuation can be applied to both types of mirrors, but coil actuation applies to type- 2 mirrors only. The following gives a detailed description of both types of actuation.

\subsection{Permalloy Actuation}

Mirror deflections (Figs. 5 and 6) for both mirror types can be produced by interaction between an external magnetic field and the magnetic moment in the NiFe layer (permalloy actuation). The external magnetic field first induces a magnetic moment in the $\mathrm{NiFe}$, and the interaction between the magnetic field and the permalloy magnetic moment produces a torque in the direction to align the moment with the field direction. In the case of a nonuniform magnetic field, a translational force that is proportional to the gradient of the magnetic field will also be produced (as shown in Fig. 7). In our experiments, though, magnetic modeling of the mirror actuation is investigated in a uniform magnetic field, $\mathbf{H}_{\text {ext }}$. The magnetic field is perpendicular to the wafer surface, and an out-of-plane rotation of the plate is induced. The deflection angle for each mirror is then measured at different field strengths and is shown in Fig. 8.

The deflection for both mirrors is modeled using the torque balance (Fig. 9) between the permalloy torque ( $\left.T_{\mathrm{NiFe}}\right)$ and the restoring torque of the spring $\left(T_{\text {spring }}\right)$. The permalloy torque $T_{\mathrm{NiFe}}$ (also described in the literature ${ }^{3,4}$ ) is produced by the interaction between $\mathbf{B}_{\text {ext }}$ and a magnetic moment $\mathbf{m}_{\mathrm{NiFe}}$ in the permalloy:

$T_{\mathrm{NiFe}}=\left|\mathbf{m}_{\mathrm{NiFe}} \times \mathbf{B}_{\mathrm{ext}}\right|=V_{\mathrm{NiFe}} M_{\mathrm{NiFe}} H_{\mathrm{ext}} \sin (90 \mathrm{deg}-\theta)$,

where $V_{\mathrm{NiFe}}=7.26 \times 10^{-11} \mathrm{~m}^{3}$ is the volume of the permalloy, $H_{\text {ext }}$ is given in amperes per meter, $\theta$ is the deflection angle of the flap relative to the substrate, and $M_{\mathrm{NiFe}}$ is the magnetization of the permalloy induced by $H_{\text {ext }} \cos (90 \operatorname{deg}-\theta)$. The torque (in newton meters) is then counterbalanced by the restoring torque of the spring as

$T_{\text {spring }}=K \theta$,

where $K=K_{1}=9.2 \times 10^{-8} \mathrm{~N} \mathrm{~m} / \mathrm{deg}, \quad K=K_{2}=4.2 \times 10^{-8}$ $\mathrm{Nm} / \mathrm{deg}$ are the measured torsional spring constants for the type- 1 and type- 2 mirrors, respectively, and $\theta$ is the deflec- 

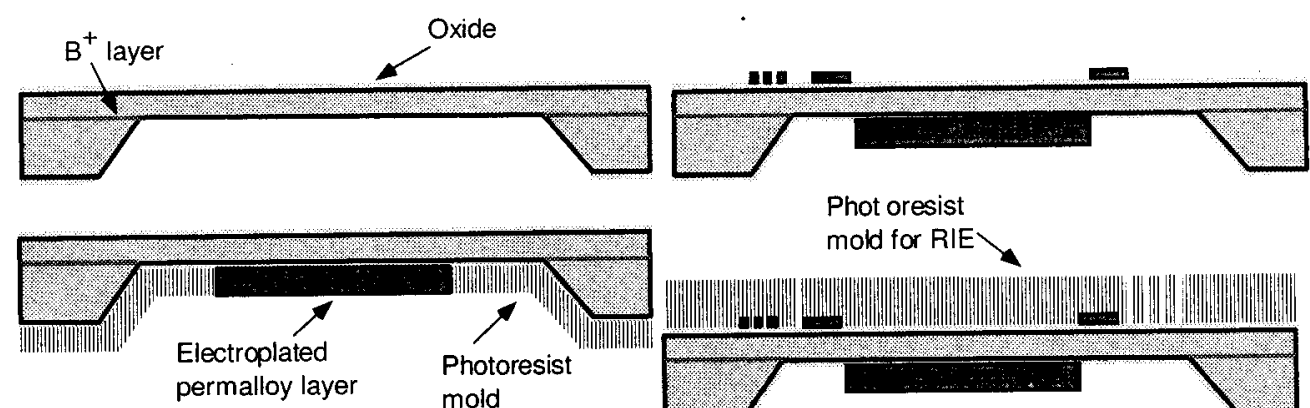

Phot oresist mold for RIE
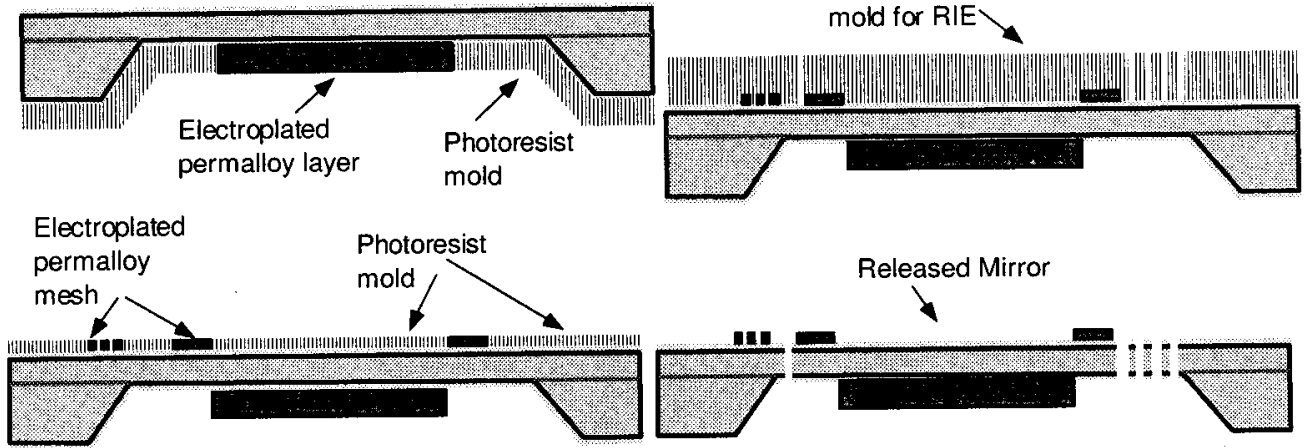

Fig. 3 Schematic showing key type-1 mirror fabrication steps.

tion angle of the mirrors. Equations (1) and (2) are the governing equations for the operation of the mirrors as shown in Fig. 8, where the theory (solid and dashed lines) fits the experimental data very well. Note that the necessary information on $M_{\mathrm{NiFe}}\left(H_{\text {ext }}\right)$ was determined experimentally by $B H$-loop and vibrating-sample magnetometer (VSM) measurements performed on the permalloy layer of each mirror. The $B H$ loop gives the shape of $M_{\mathrm{NiFe}}\left(H_{\mathrm{ext}}\right)$, while the VSM measurement determines the saturated magnetization $\left(M_{s}\right),{ }^{5}$ which is $0.79 \mathrm{~T}$ for the type- 1 mirrors and 0.78 $\mathrm{T}$ for the type- 2 mirrors.

\subsection{Coil Actuation}

Coil actuation comes from the interaction between an external magnetic field and an electrical coil with a flowing current. The type- 2 mirrors can be operated in this coilactuation mode, where an applied current to the on-plate coils (in the presence of a magnetic field) can control the mirror deflection angle. For type- 2 mirrors, a constant external magnetic field will first cause a permalloy flap to deflect to a fixed "bias" angle due to the permalloy actuation. Deflection about this position can then be further controlled by changing the magnitude and direction of the cur-
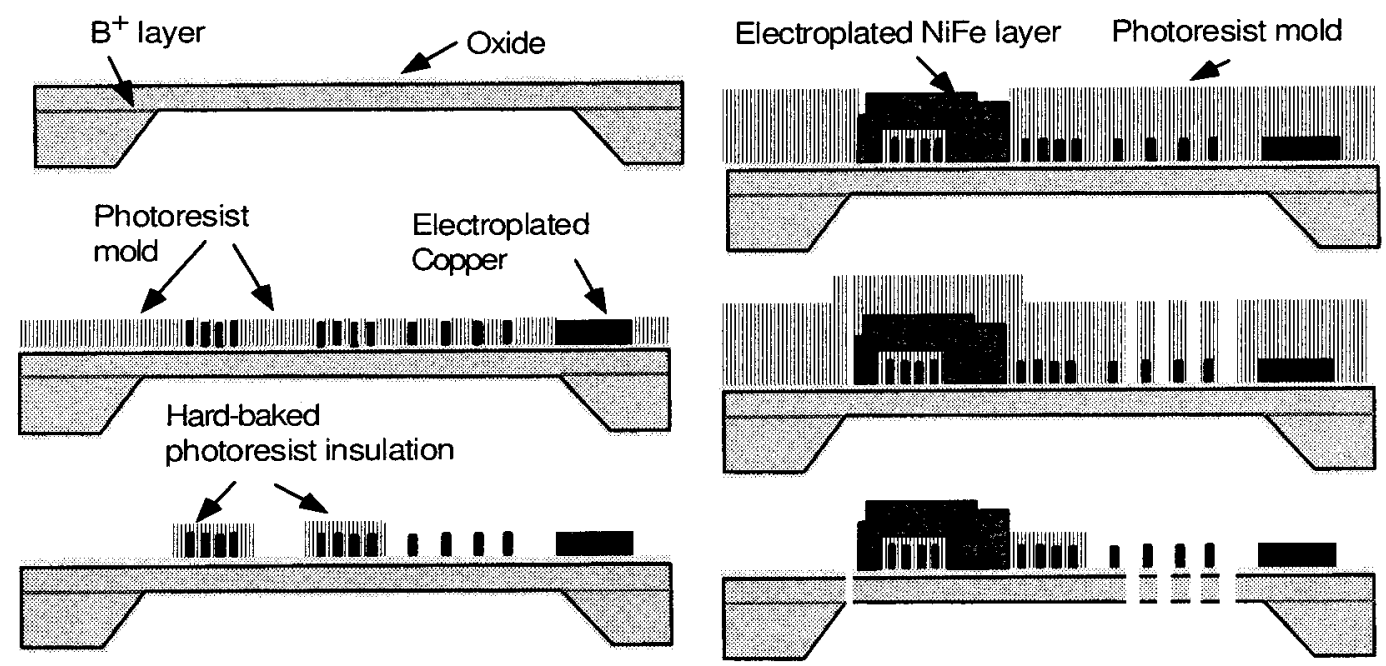

Fig. 4 Schematic of type-2 mirror fabrication steps. 


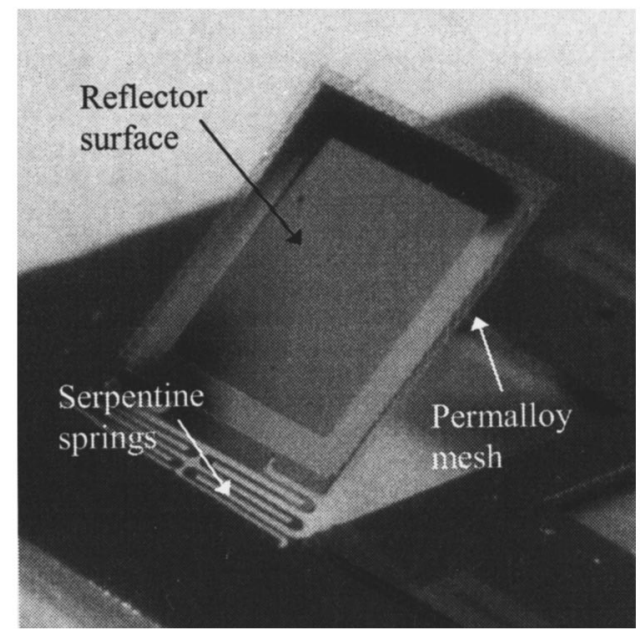

Fig. 5 Photograph of actuated type-1 MEMS mirror.

rent supplied to the coils (Fig. 10). A convenient way of controlling a type- 2 mirror is then to use the external field for coarse deflection control and to use the coil current for fine positional control. This operation has been explored experimentally, and Fig. 11 shows the coil-actuated deflection angle of a type-2 mirror in an external bias field set to $79 \mathrm{kA} / \mathrm{m}$ (994 Oe). Without the coil current, this magnetic field saturates the magnetic moment in the permalloy and then causes a bias angle $\theta=58 \mathrm{deg}$ due to permalloy actuation.

To model the coil actuation (as in Fig. 11), we add two more terms, $T_{\text {coil }}$ and $T_{\text {thermal }}$, in the torque balance equation (as shown in Fig. 12). The $T_{\text {coil }}$ term is the torque induced by a current moment, $\mathbf{m}_{\text {coil }}=I A_{\text {total }}$, in a magnetic field given as

$T_{\text {coil }}=\left|\mathbf{m}_{\text {coil }} \times \mathbf{B}_{\text {ext }}\right|=m_{\text {coil }} B_{\text {ext }} \sin \theta$,

where $I$ is the current supplied to the coils in amperes, $A_{\text {total }}=0.000132 \mathrm{~m}^{2}$ is the sum of 30 turn areas, and

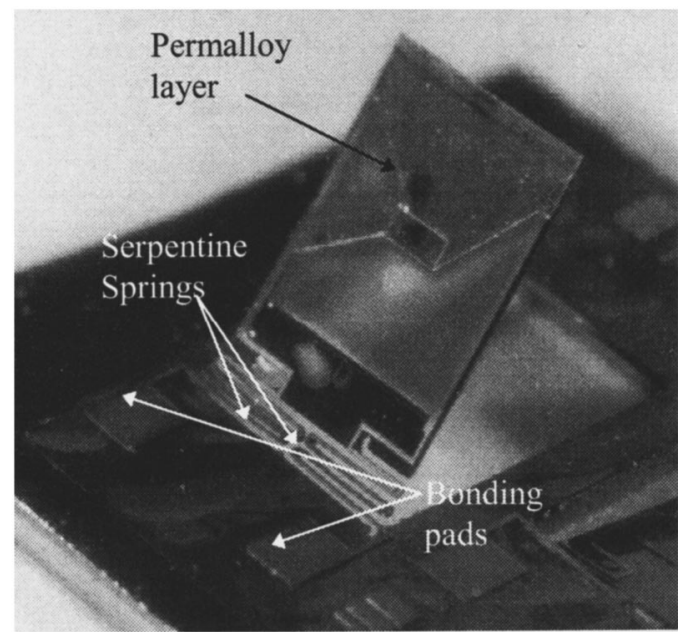

Fig. 6 Photograph of actuated type-2 MEMS mirror.
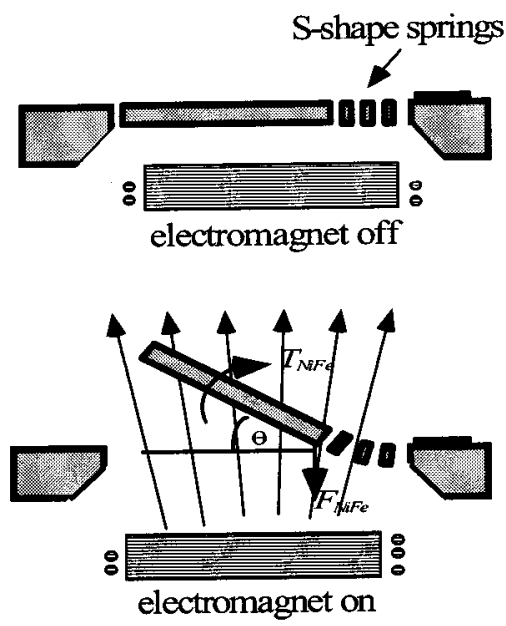

Fig. 7 Schematic of mirror actuation concept.

$B_{\text {ext }}=\mu_{0} H_{\text {ext }}$ is the magnetic flux density in teslas. On the other hand, the thermal torque is induced by heat generated by this current flowing in the coils. This is modeled by

$T_{\text {thermal }}=-C I^{2}$.

In this case $C=25.2 \times 10^{-6} \mathrm{~N} \mathrm{~m} / \mathrm{A}^{2}$ is a fitting parameter, while $I$ is the applied coil current in amperes. The negative sign in Eq. (4) means that the thermal torque always bends the mirror down. As a result, when a current is applied to the coils with a constant external magnetic field, the torque balance equation becomes

$V_{\mathrm{NiFe}} M_{s} H_{\mathrm{ext}} \cos \theta+m_{\mathrm{coil}} B_{\mathrm{ext}} \sin \theta-C I^{2}=K \theta$.

Figure 11 then shows both the experimental and theoretical data on a type- 2 mirror controlled by the coil actuation. The theory is shown with the solid line, and a good fit is obtained. Note that Fig. 11 also shows that the coil actuation can produce a $\pm 10-\mathrm{deg}$ deflection, which is useful for scanning-mirror applications.

\section{Mirror Quality}

For the type-1 mirror, the actuating permalloy layer is located on the bottom side of the plate. We design it this way so the original silicon top surface is used as the mirror surface with an excellent surface roughness, less than 10 $\mathrm{nm}$ over the entire mirror. However, the slight stress in the permalloy layer does cause the mirror to become slightly convex. This curvature actually depends on the magnetic field. While the measured horizontal radius of curvature is a constant $7.5 \mathrm{~cm}$, the vertical radius of curvature varies from 20 to $25 \mathrm{~cm}$, corresponding to a magnetic field of 0 to 20 $\mathrm{kA} / \mathrm{m}$. For thinner permalloy layers the radius of curvature becomes larger $(>75 \mathrm{~cm}$ for 5 - $\mu \mathrm{m}$-thick permalloy) but does not change with field strength.

For the type- 2 mirror, the top side of the mirror is used to place the permalloy and coils, so the bottom side of the silicon plate is the reflector. The bottom surface is slightly rougher $(\approx 45 \mathrm{~nm})$, because we used an epitaxy wafer in our process. It is found that this mirror is also convex, with 


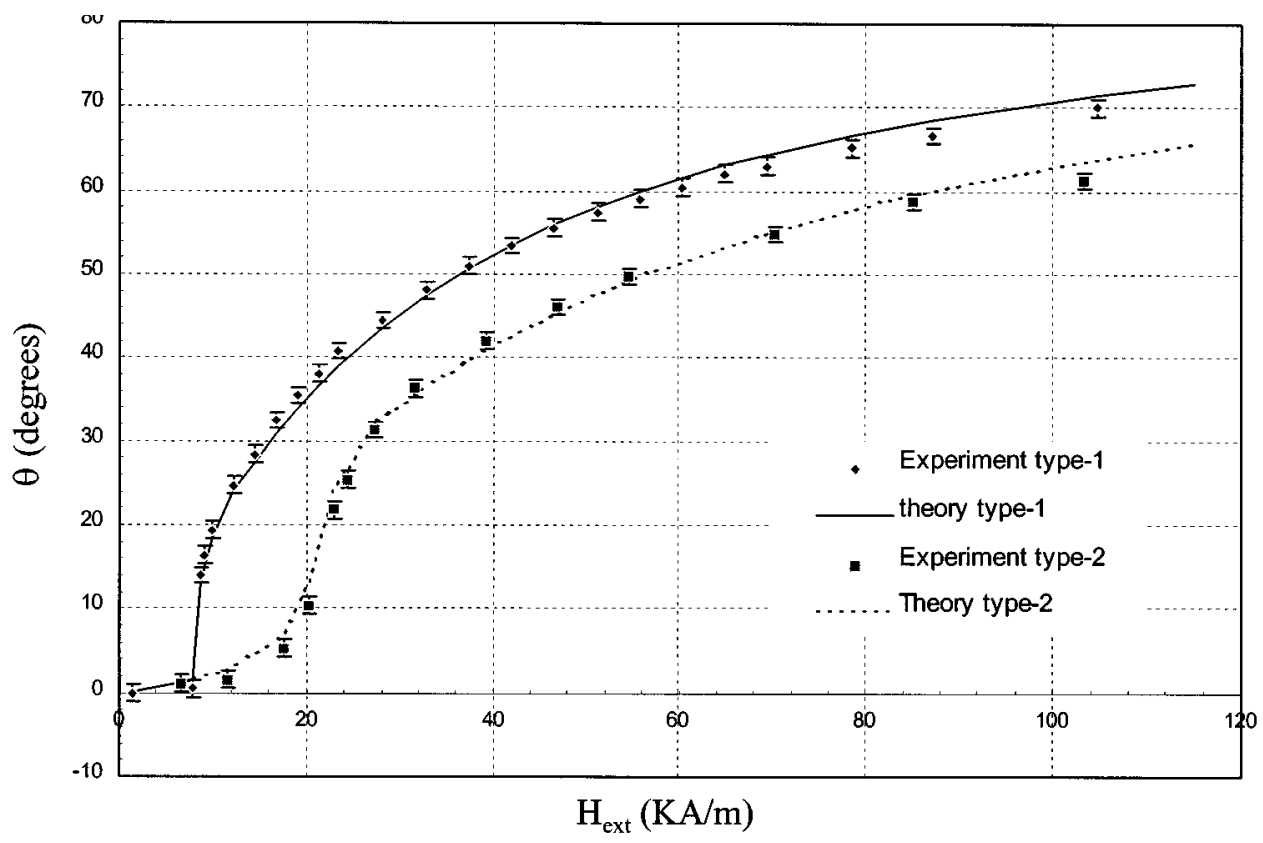

Fig. 8 Deflection angle of type-1 and type-2 MEMS mirrors versus external magnetic field.

a curvature radius of $1 \mathrm{~cm}$. Interestingly, though, we observed no change in radius of curvature at field strengths up to $23 \mathrm{kA} / \mathrm{m}$ (300 Oe). Nevertheless, if necessary, the surface roughness and the warpage of the mirrors can both be easily improved by using silicon-on-insulator (SOI) wafers. For our use of the mirrors in laser holographic data storage, the imperfection of the mirror is not important. As long as the reflected beam off the mirror for a given angle is consistently reproducible, good holograms can be written and retrieved, as demonstrated in the following.

\section{Holographic Data Storage}

In general, volume holographic data storage is achieved by using two laser beams (one reference beam and one signal beam), which interfere with each other and form a 3-D interference pattern (hologram) in a light-sensitive crystal (such as $\mathrm{Fe}$-doped $\mathrm{LiNbO}_{3}$ ) that "memorizes", it. In this process, the interfering beams change the optical properties

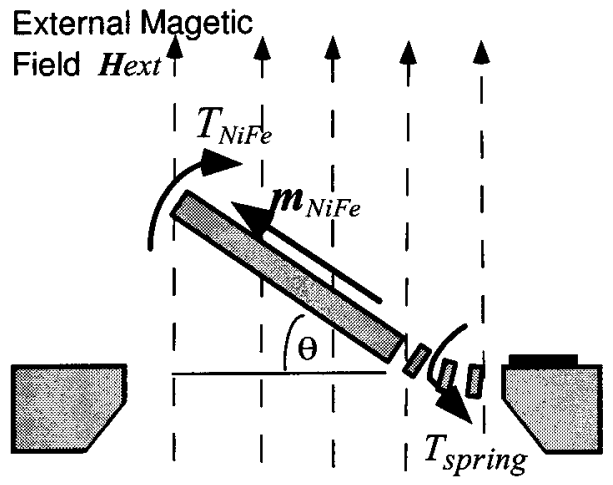

Fig. 9 Torque balance of the mirror with permalloy only, in an external magnetic field. of the crystal, and the crystal stores this 3-D interference grating. To read the data, only the reference beam is needed to shine through the crystal, and the prestored 3-D grating inside the crystal reproduces the signal beam. ${ }^{6}$ Holographic memory systems with both large capacity and rapid access require high-speed wide-angle beam-steering elements. Mechanically rotated conventional mirrors or acousto-optic deflectors were commonly used in experiments. These devices are bulky and expensive, and they introduce noise through either pointing inaccuracy or wavefront distortion. An emerging deflector technology is based on liquid crystals. However, devices with a large number of deflection angles are difficult to fabricate. Here we report the results on the first use of MEMS mirrors in holographic storage. These devices potentially offer low cost, low mass, and large scanning angles.

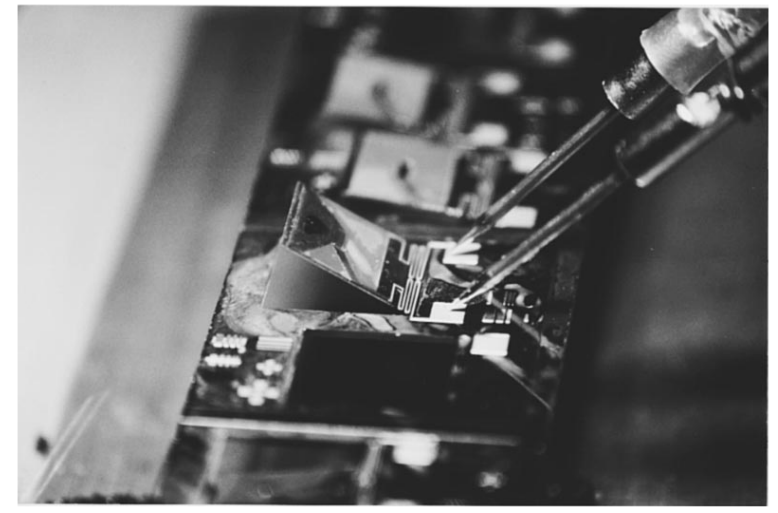

Fig. 10 Actuated mirror in an external field, $\left[H_{\text {ext }}=23.9 \mathrm{kA} / \mathrm{m}(300\right.$ $\mathrm{Oe}$ ) perpendicular to the substrate], with a coil current of around 30 $\mathrm{mA}$. The deflection is caused by both the permalloy and the coil current. 


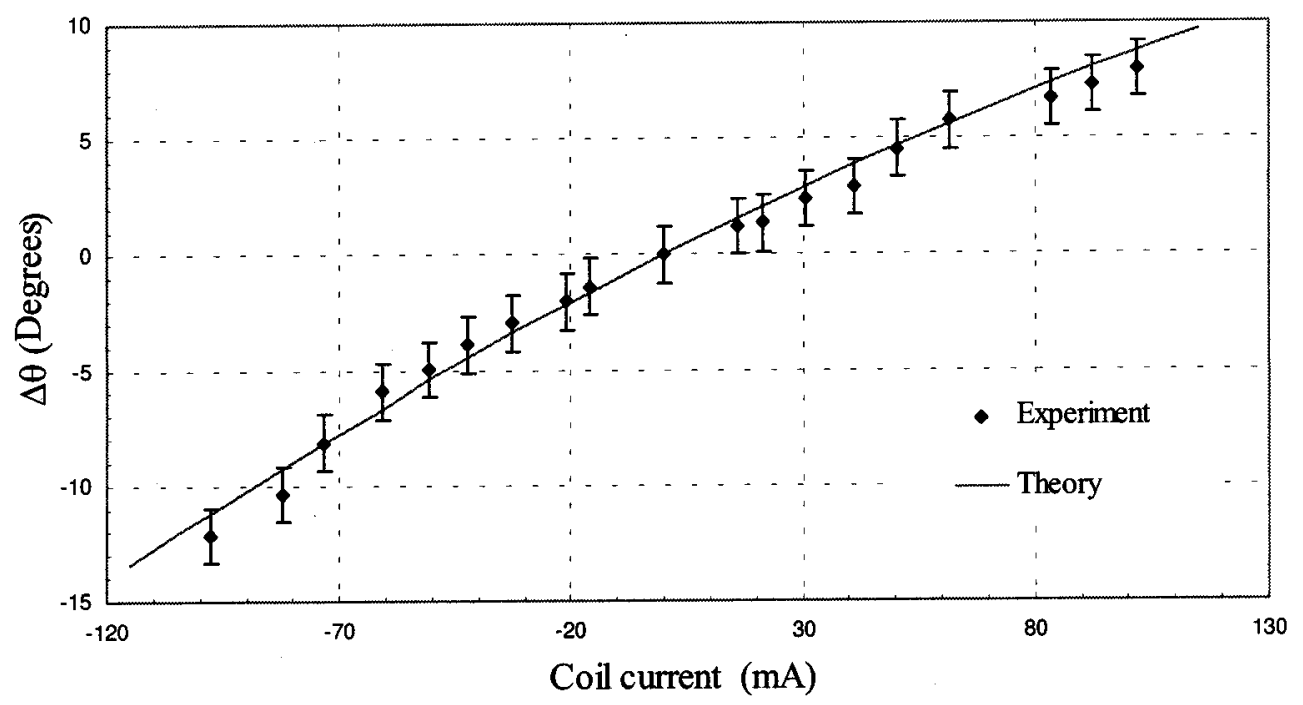

Fig. 11 Change in deflection angle from bias position for variable coil current with external field of 79 $\mathrm{kA} / \mathrm{m}(994 \mathrm{Oe})$.

\subsection{Type-1 MEMS Mirror}

Using the setup shown in Fig. 13(a), we demonstrated the type-1 mirror as a deflector for angle-multiplexed holographic storage. The mirror was placed on a solenoid-type electromagnet, which was driven by a dc voltage regulator to generate the desired magnetic field. In this system configuration a single laser beam (argon laser) is split into a reference beam and a signal beam path. The latter contains an information presentation device (liquid-crystal display), a storage crystal, and a detector (CCD camera). The reference beam is deflected by the MEMS mirror and then passes through a lensing system. The lenses keep the beam focused on the same location on the crystal but with different incident angles corresponding to the mirror deflection angle. The volume nature of the holograms allows a page of data to be stored for each different deflection angle, provided that these angles are sufficiently separated. In this demonstration, we stored 100 holograms using the type- 1

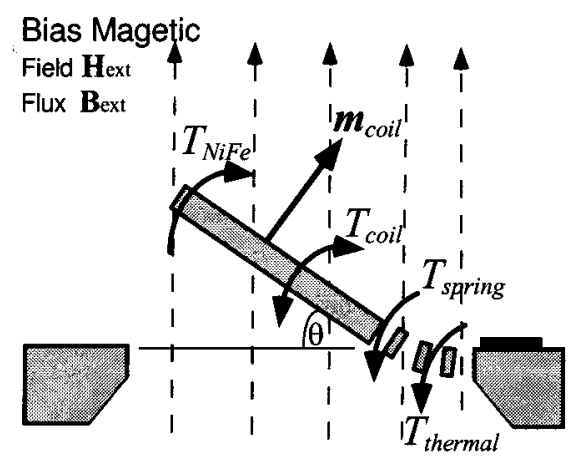

Fig. 12 Torque balance of the mirror with both the permalloy and the coil current in an external magnetic field. mirror. Some hologram reconstructions are shown in Fig. 14. The holograms are of good quality except for the conspicuous concentric circles, which are an artifact of the crystal surface not being polished well. Although each hologram was spaced from its neighbor by $0.143 \mathrm{deg}$, much smaller deflections $(0.007 \mathrm{deg})$ could be generated with the mirror and the control circuit used. Practically, many more holograms can be stored than the 100 we have demonstrated.

In this experiment we found that to improve the mirror response time the mirror should be deflected in small angular steps by incrementally providing additional current to the electromagnet until reaching the desired mirror deflection angle. These small angle increments minimize the overshoot and produce an overall faster mirror response. As an example, a deflection of $10.7 \mathrm{deg}(0.187 \mathrm{rad})$, incremented in steps of $0.7 \mathrm{deg}(0.012 \mathrm{rad})$, could be performed in $\approx 200 \mathrm{~ms}$. If the 10.7-deg jump was executed in one step, the mirror would ring for more than $400 \mathrm{~ms}$. We also investigated how accurately the mirror could return to a specific deflection angle. The type-1 mirror was deflected from 0 to 53 deg more than 20 times. Each time we found that the mirror returned to within $0.003 \mathrm{deg}(64 \mu \mathrm{rad})$ of the 53-deg deflection angle. Finally, the angular stability of the mirrors was also measured when the mirror was deflected to $53 \mathrm{deg}$; any change from the deflection angle over a 5-min period was noted. The measured angular fluctuation of this mirror was less than $0.0006 \mathrm{deg}(10 \mu \mathrm{rad})$.

\subsection{Type-2 MEMS Mirror}

In the holographic data storage demonstration using the type-2 mirror, the mirror was first biased to approximately $45 \mathrm{deg}$ in a constant but nonuniform external field $(\approx 200$ Oe at the mirror $)$, provided by a permanent magnet located $2 \mathrm{~mm}$ above the surface of the mirror. Deflections about this angle (fine control) were induced by varying the coil current. Two multiplexing schemes were explored. First the type- 2 mirror was used in the same angle- 


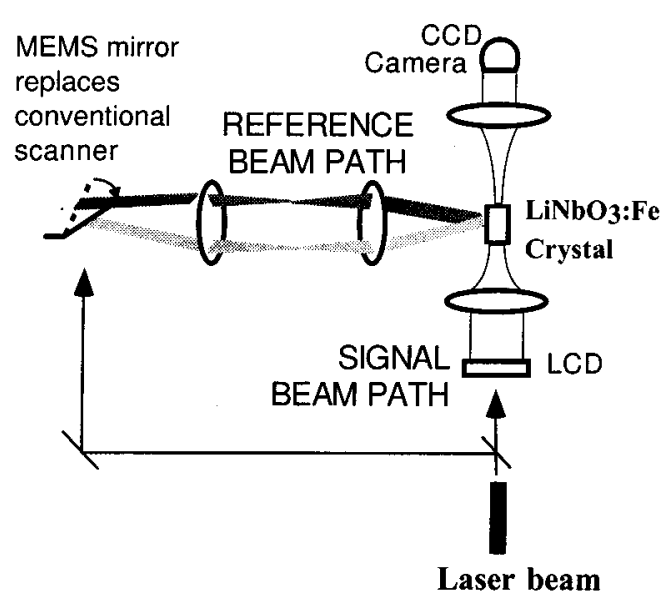

(a)

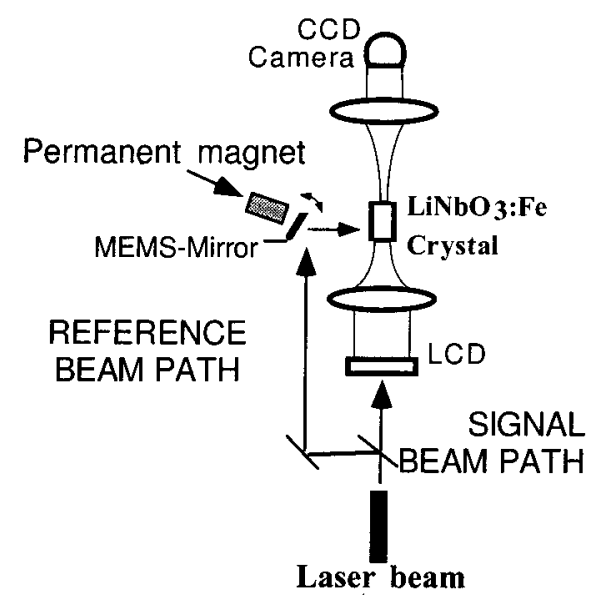

(b)

Fig. 13 (a) Conventional holographic storage system using commercial beam deflectors. (b) Compact storage system with MEMS mirror.

multiplexing storage system that the type-1 mirror was used in [Fig. 13(a)]. The same success was obtained, and Fig. 15 shows some typical holograms stored and then retrieved using the MEMS scanning mirror. In addition to the angular multiplexing scheme, the mirror was also used in a combined spatial/angular multiplexing configuration [Fig. 13(b)]. Here, not only was the incident angle of the reference beam changed, but so was its position on the crystal. The primary advantage of this configuration is that two lenses can be eliminated and the mirror placed right at the crystal surface. Once again, for demonstration purposes we stored 100 holograms; all were of good quality.

Fixed angular increments of $\approx 0.3 \mathrm{deg}(5 \mathrm{mrad})$, for the type-2 mirror, were found to provide the shortest time to reach and settle on a particular hologram. Each 0.3-deg angle increment took $\approx 25 \mathrm{~ms}$. In future work we plan to investigate other methods to improve the mirrors' response. Some possibilities include increasing the mechanical damping of the springs by coating them with lossy materials such as polyimide, increasing the spring stiffness, or shaping the driving input signals so as not to excite resonant modes. The type-2 mirror (magnetic field fixed and current supplied to the coils) returned to within $0.002 \mathrm{deg}(40 \mu \mathrm{rad})$ when the mirror was deflected many times, in this case from 0 to $2 \mathrm{deg}$. Once the mirror was deflected to the above deflection angle, its angular stability was also measured. Any change from the above deflection angle over a 5-min period was noted. The angular fluctuation for this mirror was less than $0.0006 \mathrm{deg}(10 \mu \mathrm{rad})$.
As a conclusion, the MEMS mirrors are an attractive angle-multiplexing technology for holographic data storage. They possess a very large continuous deflection range, are compact, and use only moderate power. Though the two types of mirrors performed equally well in their ability to store and retrieve holograms, in terms of operational versatility the type- 2 mirror may be more advantageous.

\section{Discussion}

From a technological point of view, several new issues arise from the incorporation of magnetic thin-film materials into silicon bulk micromachining. Magnetic forces in microactuators are proportional to the volume of magnetic material. Therefore, microactuators generally require thicker layers of magnetic material than those used in thinfilm recording heads if a large magnetic force is desired. This, however, introduces stress and adhesion problems. Also, care must be taken that the thermal cycling and chemical etching used in the micromachining process does not damage the magnetic materials. Finally, magnetic properties such as the remanent magnetization, saturation field, and coercivity of the films may need to be optimized for the specific requirements of the device.

For evaluation purposes a comparison of the MEMS mirrors with some commercial scanning devices is provided in Table 1. Although the indicated hologram spacing for the commercial devices is better than for the MEMS mirrors, the objective has been simply to demonstrate that
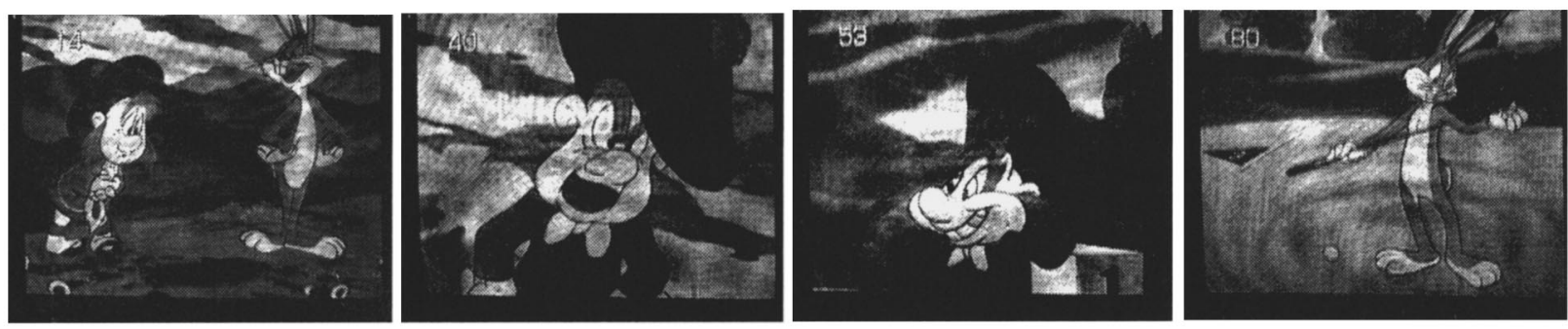

Fig. 14 Four random holograms stored and retrieved using the type-1 mirror. 


\section{Original "Signal" beam}
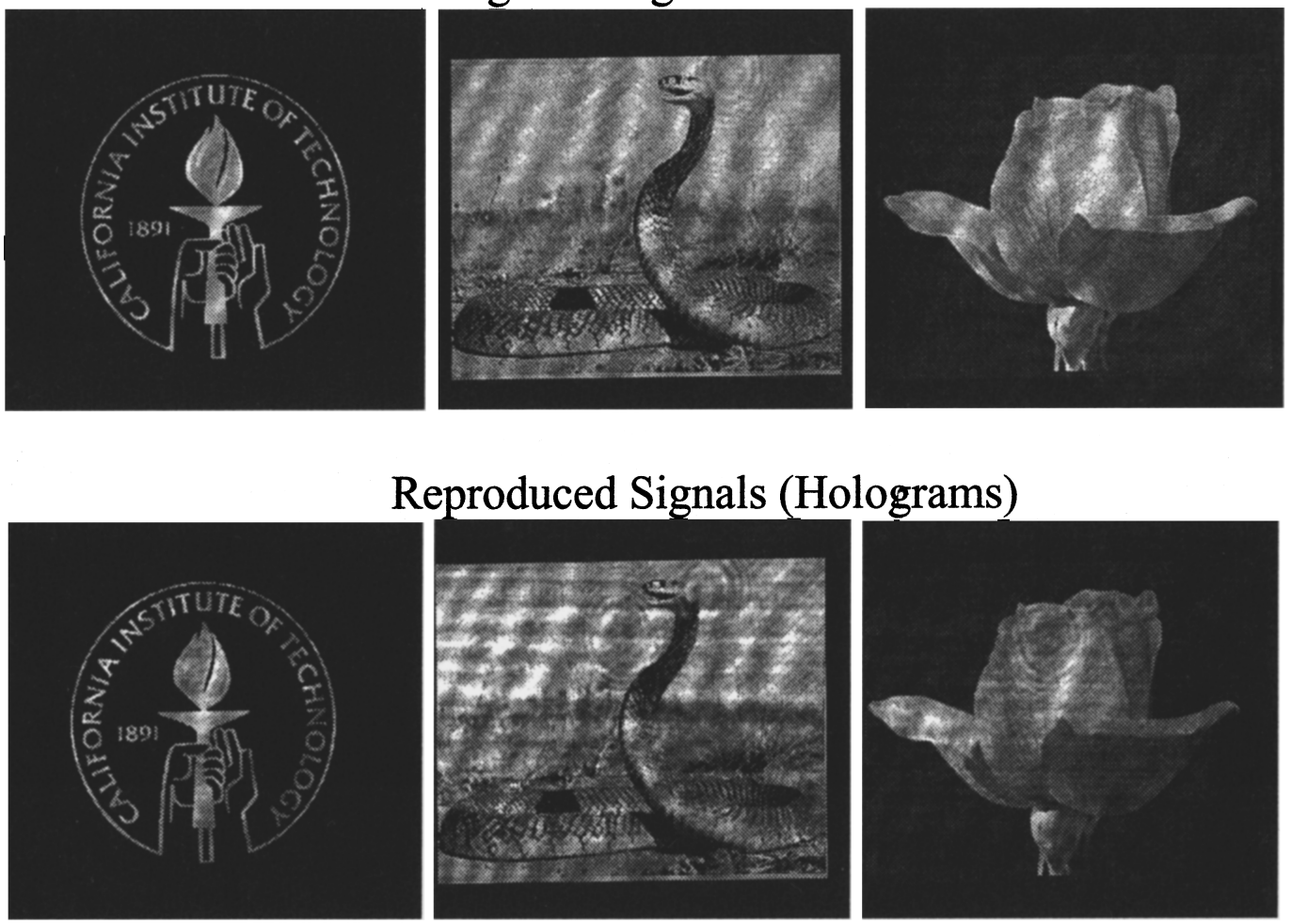

Reproduced Signals (Holograms)

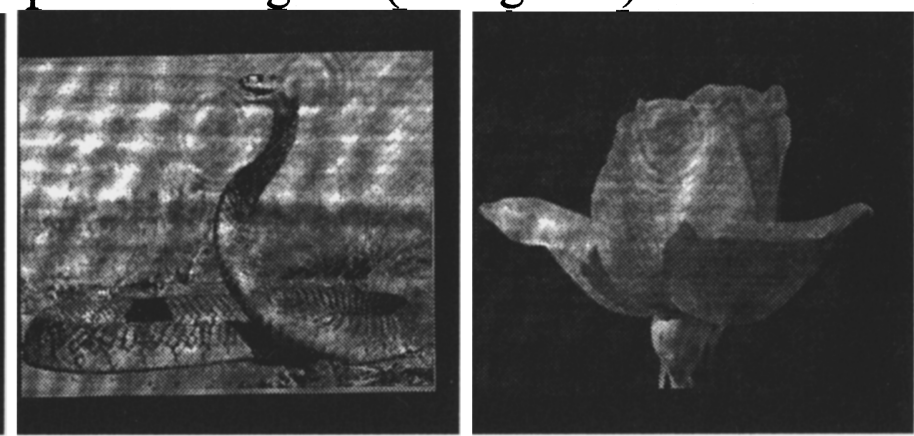

Fig. 15 Holograms stored and retrieved, showing original image prior to storage, and retrieved image from crystal.

the storage of $\sim 100$ holograms using the MEMS mirrors is practical. Future work is planned to determine how closely, in angle, these holograms can be spaced using the MEMS mirrors. On the other hand, the deflection range for both MEMS mirrors is larger than for either of the commercial devices. To reduce thermal effects due to current heating in the type-2 mirrors, the current-induced deflection range of $20 \mathrm{deg}$ can be reduced while incorporating the external coarse control. The time response of the MEMS mirrors is significantly better than that of the commercial motorized stage, but worse than that of the acousto-optic deflector.
Mirror response times can be further improved by increasing the resonant frequency of the mirrors (compromising some of the large deflection range) or increasing mechanical damping as mentioned previously. From Table 1 it is apparent that using the MEMS mirror does significantly reduce the overall volume of the demonstrated holographic storage system. To further improve storage capacity, future work may include a design for a multi-MEMS-mirror array storage system.

Table 1 Comparison of MEMS mirror with two commercial scanning devices.

\begin{tabular}{|c|c|c|c|c|c|c|}
\hline Device & $\begin{array}{c}\text { Achieved } \\
\text { hologram } \\
\text { spacing } \\
\text { (deg) }\end{array}$ & $\begin{array}{l}\text { Deflection } \\
\text { range } \\
\text { (deg) }\end{array}$ & $\begin{array}{l}\text { Access } \\
\text { time for } \\
0.3-\text { deg } \\
\text { deflection } \\
\text { (ms) }\end{array}$ & $\begin{array}{l}\text { Reference- } \\
\text { beam path } \\
\text { volume } \\
\left(\mathrm{cm}^{3}\right)\end{array}$ & $\begin{array}{l}\text { Device } \\
\text { volume } \\
\left(\mathrm{cm}^{3}\right)\end{array}$ & Cost \\
\hline \multicolumn{7}{|l|}{ MEMS: } \\
\hline Type-1 mirror & 0.143 & 60 & 33 & 100 & 5 & Potentially low \\
\hline Type-2 mirror & 0.03 & 60 & 25 & $1-10^{\mathrm{b}}$ & 1 & Potentially low \\
\hline $\begin{array}{l}\text { Commercial } \\
\text { motorized } \\
\text { stage }\end{array}$ & 0.002 & 35 & 130 & 686 & 60 & High \\
\hline $\begin{array}{c}\text { Acousto-optic } \\
\text { deflector }\end{array}$ & 0.0015 & 1.5 & 0.070 & 1372 & 30 & High \\
\hline
\end{tabular}

${ }^{\mathrm{a} N o t}$ including laser.

${ }^{\mathrm{b}}$ For compact configuration as in Fig. 13(b). 


\section{Conclusion}

Large-angle-of-deflection scanning MEMS mirrors have been successfully developed. The operation of mirrors with permalloy only and mirrors combining permalloy and coils have been modeled, and good agreement is found between theory and experiment. Significant reduction in holographic system volume is achieved using the mirrors. Finally, the mirrors have been successfully demonstrated in a holographic storage application, demonstrating the feasibility of combining bulk micromachining with magnetic thin-film actuation technology.

\section{Acknowledgment}

This project was partially supported by AFOSR and DARPA under AFOSR Grant No. F49620-94-1-0008. The authors would like to thank Geoffrey Burr, Gregory Billock, and Dr. Demetri Psaltis for using the MEMS mirrors in their holographic storage systems. Without their help this work would not be possible. We also thank Gordon Hughes of Seagate technology, and Stanley Bacon for their assistance with magnetic measurements.

\section{References}

1. J. Bryzek and K. Petersen, "Micromachines on the march," IEEE Spectrum, 31 (5), 20-31 (May 1994).

2. C. Liu, T. Tsao, Y. C. Tai, "A micromachined permalloy magnetic actuator array for micro robotics assembly systems," in Proc. 8th International Conference on Solid-State Sensors and Actuators (Transducers 95), Vol. 1, pp. 328-331, Royal Swedish Academy of Engineering Sciences, IVA, and the 1995 Foundation for Sensor and Activator Technology, Stockholm, Sweden (1995).

3. J. W. Judy and R. S. Muller, "Magnetic microactuation of torsional polysilicon structures," in Proc. 8th International Conference on Solid-State Sensors and Actuators (Transducers 95), Vol. 1, pp. 332335, Royal Swedish Academy of Engineering Sciences, IVA, and the 1995 Foundation for Sensor and Activator Technology, Stockholm, Sweden (1995)

4. C. Liu, T. Tsao, Y. C. Tai, J. Leu, T. S. Leu, C. M. Ho, W. L. Tang, and D. Miu, "Out-of plane permalloy magnetic actuators for deltawing control,' in Proc. IEEE International Workshop on Micro Electro Mechanical Systems (MEMS 95), pp. 328-331 (1995).

5. R. A. Miller, G. W. Burr, Y. C. Tai, D. Psaltis, C. M. Ho, and R Katti, "Electromagnetic MEMS scanning mirrors for holographic data storage," in Proc. Solid-State Sensor and Actuator Workshop, pp. 183-186, Transducers Research Foundation, Inc., Hilton Head, SC (1996).

6. D. Psaltis and F. H. Mok, "Holographic memories," Sci. Am. 273(5), 70-75 (1995).

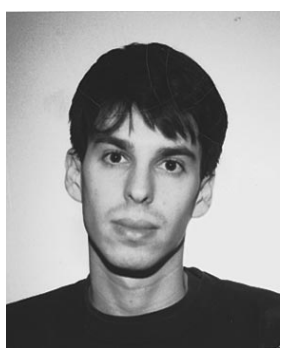

Raanan A. Miller received his BS degree in electrical engineering from Boston University in 1990, and his MS from the California Institute of Technology in 1992. Currently he is completing his $\mathrm{PhD}$ at Caltech developing electromagnetic MEMS actuators.

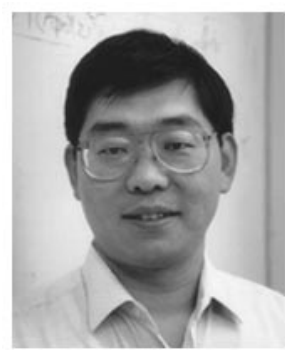

Yu-Chong Tai received his $\mathrm{PhD}$ in electrical engineering from the Department of Electrical Engineering of the University of California at Berkeley in 1989. After Berkeley, he joined the faculty of electrical engineering at the California Institute of Technology (Caltech), where he is currently an associate professor. Dr. Tai's major research since his PhD has been micromachining for solid-state microsensors and microactuators (or MEMS). At Berkeley, he was the inventor of the first electrically spun polysilicon micromotor. At Caltech, he has built a micromachining laboratory and a program of about 15 researchers. His current research interests include new MEMS technologies and devices such as shearstress sensors, pressure sensors, culture neuron probes, micro fluidic devices, micromotors for hard-disk drives, microphones, magnetic microactuators, and MEMS systems for active fluid control. Because of his research, he has received several awards, such as the IBM fellowship, the Best Thesis Award, the presidential Young Investigator (PYI) Award, and the David and Lucile Packard Fellowship. He is also a member of the editorial board of the Journal of Micromechanics and Microengineering. 